\title{
Secondary Raynaud's Phenomenon and Skin Necrosis of Toes in the Paraplegic Patient with Hypertension
}

\author{
Yong Jig Lee ${ }^{1}$ (D) Kisoo Park ${ }^{1}$
}

Published online: 7 February 2018

(C) The Author(s) 2018. This article is an open access publication

\begin{abstract}
We present two cases of paraplegic patients who developed secondary Raynaud's phenomenon. A 43-yearold man with paraplegia presented with dark purple discoloration and skin defects on his left second and third toes and complained of a cold sensation in both feet for a period of 1 year. He had been taking diuretics for 4 years. The capillary refilling time for both affected toes was delayed. His antihypertensive drug was changed to a calcium channel blocker under suspicion of Raynaud's phenomenon aggravated by hydrochlorothiazide, and the capillary refilling time normalized within 3 days. The toe skin defect was covered with a skin graft. A 51-year-old man with paraplegia presented with cyanotic color change and recurrent unstable wounds on his toes. He was also taking diuretics for hypertension. Suspecting secondary Raynaud's phenomenon aggravated by diuretics, we changed the diuretics to olmesartan medoxmil $20 \mathrm{mg}$ and amlodipine besylate $2.5 \mathrm{mg}$ per day. Subsequently, he has had no unstable wounds for 30 months. If hypertensive patients with paraplegia complain of skin discoloration in their extremities, Raynaud's phenomenon should be considered and the antihypertensive drug may need to be stopped in order to improve the wound-healing process.
\end{abstract}

Yong Jig Lee

syjlee@cu.ac.kr

1 Department of Plastic and Reconstructive Surgery, Daegu Catholic University, School of Medicine, (\#42472) 2F Raphael Building, 33 Duryugongwon-ro 17-gil, Nam-gu, Daegu, South Korea

\section{Key Points}

If a hypertensive patient with paraplegia complains of unstable aggravating wounds on toes, secondary Raynaud's phenomenon should be considered, especially if the patient is receiving diuretics.

Secondary Raynaud's phenomenon due to diuretics appears to be reversible on interruption of treatment.

The wounds from secondary Raynaud's phenomenon caused by diuretics may need to be operated on for wound coverage.

\section{Introduction}

Secondary Raynaud's phenomenon can develop from certain medications, especially amphetamines, $\beta$-adrenergic blockers, bleomycin, cisplatin, cyclosporine, ergots, interferon alpha, and vinblastine [1]. Although the exact mechanism or prevalence of Raynaud's phenomenon is still unclear in patients receiving diuretics, some debates and reports have been published [2]. This report details two cases of skin necrosis due to advanced secondary Raynaud's phenomenon induced by diuretics in increased peripheral vascular resistance in a paraplegic condition. 


\section{Cases}

\section{Case 1}

A 43-year-old man with paraplegia for 17 years presented with dark purple discoloration of left second and third toes beginning 3 days earlier. He complained of having a cold sensation in both feet for 1 year. He was diagnosed with hypertension 4 years earlier and was taking hydrochlorothiazide-valsartan. He had also been taking kallidinogenase, ranitidine, levosulpiride, phloroglucinol, and loxoprofen. Physical examination showed that both of the patient's feet were cold. However, the pulse was palpable on ankles and heels on both sides. A portable Doppler device (Minidop ES-100VX, Hadeco, Japan) showed that the pulse signal intensity of the left and right dorsalis pedis artery was $4-6$ and $10 \mathrm{~cm} / \mathrm{s}$ each, and that of the posterior tibial artery was $6-8$ and $10 \mathrm{~cm} / \mathrm{s}$ each. In addition, the capillary refilling time for both toes was $>10 \mathrm{~s}$. Even though a vascular function test with air-calibrated plethysmography showed a normal segmental arterial pressure ratio in thigh to ankle, normal ankle/brachial systolic index (ABI) in both ankles $(>0.9)$, and normal triphasic pulse wave pattern in thigh to ankle (Fig. 1), the skin over the left toes showed dark discoloration with skin necrosis. His antihypertensive drug was changed to a calcium channel blocker (amlodipine besylate $2.5 \mathrm{mg}$ per day) under suspicion of Raynaud's phenomenon aggravated by hydrochlorothiazide, and the cold sensation in both toes and forefeet decreased and the capillary refilling time
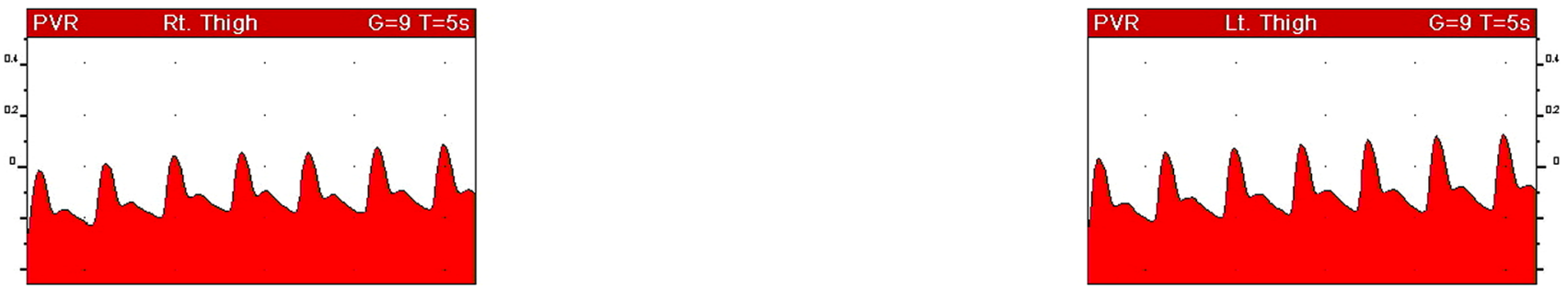
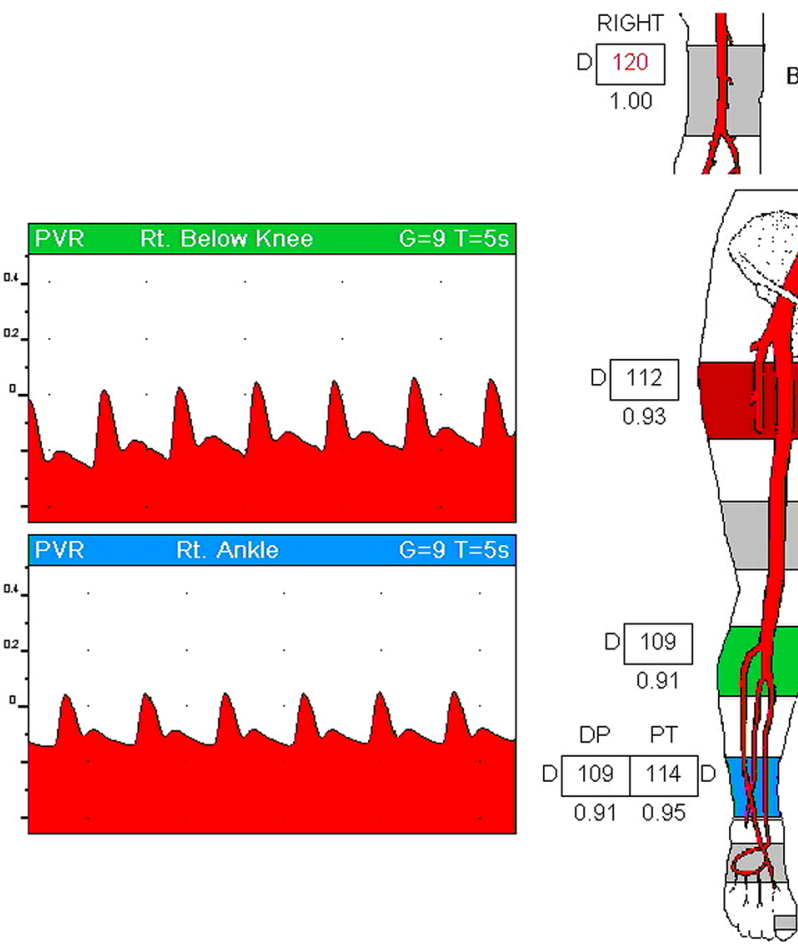

Fig. 1 Vascular function test with air-calibrated plethysmography. This is a test that checks the patient's systolic blood pressure from an upper extremity with brachial artery and lower extremity with the posterior tibial (PT) artery and dorsalis pedis (DP) artery, in a supine position. Then the ratio of the systolic pressure at the brachial artery, which is higher than the other side, to the systolic pressure at the lower extremity, which is higher between PT and DP on each side, is calculated. The ratio is called the ankle-brachial index (ABI). In this patient, the test showed a normal segmental arterial pressure ratio in thigh to ankle, normal ankle/brachial systolic index in both sides (right 0.95 , left 0.97 , normal $>0.9$ ), and normal triphasic pulse wave pattern in thigh to ankle 

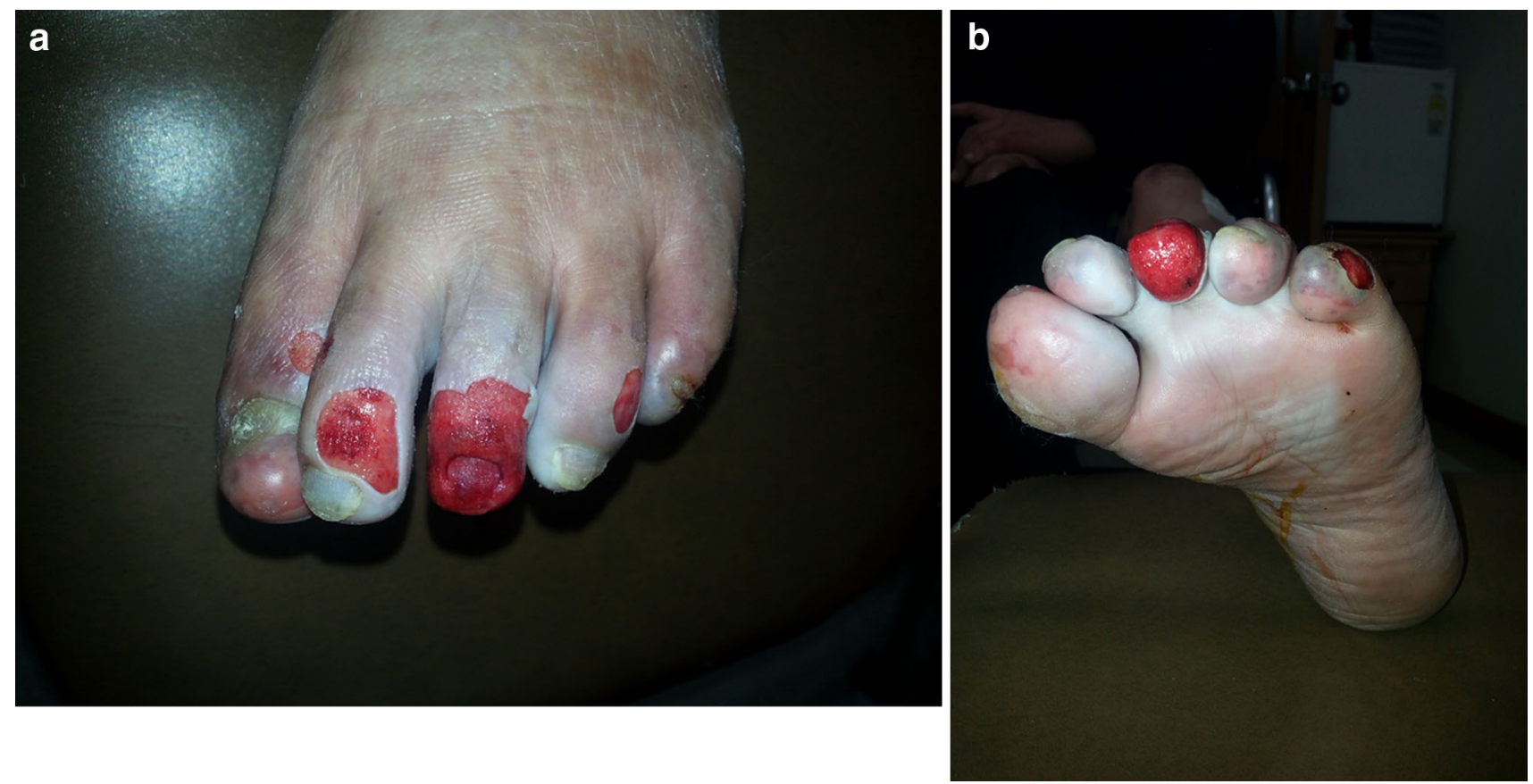

Fig. 2 Onycholysis and skin defect after advanced ischemic injury. a, b Preoperative views 7 days after starting the calcium antagonist.

avoid a small complication: skin necrosis on the left second $(1 \mathrm{~cm} \times 1 \mathrm{~cm})$ and third $(2 \mathrm{~cm} \times 1 \mathrm{~cm})$ toes. One half tablet There was no further extension of the necrotizing process with pinkcolored open wound bed. However, the progression was too fast to
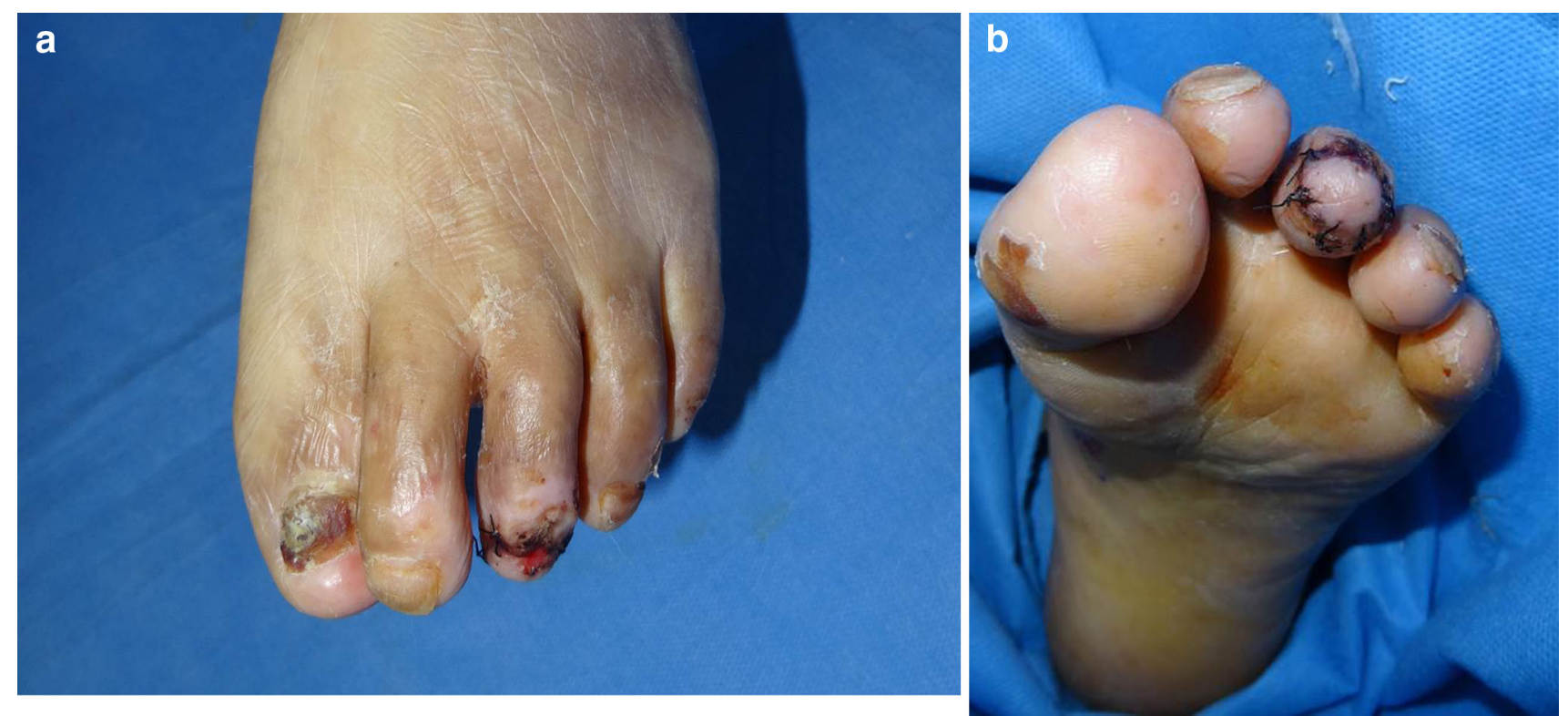

Fig. 3 Postoperative view in case 1, 9 days after operation. a, b A medium split-thickness skin graft was performed with the foot sole as a donor site

normalized within 3 days. The wound bed became well vascularized and was filled with pink granulation tissue (Fig. 2). The skin defect was too large for spontaneous healing, and a medium split-thickness skin graft was performed with the foot sole as a donor site (Fig. 3). The wound healed without complications.

\section{Case 2}

A 51-year-old man with paraplegia for 10 years presented with severe dark purple discoloration of right first and second toes, and left first, fourth, and fifth toes in October 2015. Those wounds were recurrent unstable wounds 
presenting every year from early October to late January, beginning 5 years earlier. He was diagnosed with hypertension 12 years earlier and was taking a mixture containing olmesartan medoxmil $20 \mathrm{mg}$ and hydrochlorothiazide $6.25 \mathrm{mg}$. Physical examination showed that both of the patient's feet were cold. However, a weak pulse was palpable on ankles and heels on both sides. A portable Doppler device (Minidop ES-100VX, Hadeco, Japan) showed that the pulse signal intensity of the left and right dorsalis pedis artery was 4 and $10 \mathrm{~cm} / \mathrm{s}$, respectively. In addition, the capillary refilling time for both toes was longer than $7 \mathrm{~s}$. Subsequently, his antihypertensive drug was changed to olmesartan medoxmil $20 \mathrm{mg}$ and amlodipine besylate $2.5 \mathrm{mg}$ per day under suspicion of Raynaud's phenomenon aggravated by hydrochlorothiazide. The cold sensation in both toes and forefeet did not decrease; however, the capillary refilling time was normalized to $<2 \mathrm{~s}$ within 7 days. He has not had any unstable wounds so far for 30 months.

\section{Discussion}

Raynaud's phenomenon is an episodic vasospastic disorder of peripheral arteries and arterioles, usually in response to cold and emotional triggers [3]. Mediators such as catecholamines and endothelin-1, 5-hydroxytryptamine are released in response to cold and can cause digital artery vasoconstriction and symptoms of Raynaud's phenomenon [4]. Raynaud's phenomenon is characterized by a threephase color change of blanching, cyanosis, and rubor in distal extremities after exposure to cold and subsequent rewarming. It is difficult to observe the triphasic color change even in winter because most patients wait in a warm waiting room long enough for any color change to disappear [5]. In patients with Raynaud's phenomena, approximately $60 \%$ show this typical triphasic color change, 14-40\% show two phases in color change, and 10-30\% show one phase only [6]. In this case, the patients still had cold feet with no color change, seemingly fixed in the cyanosis state. They had a minimal response to warming-up with a blanket, and had very slow and incomplete color change from cyanosis to rubor during the capillary refilling test. After spinal-cord injury, centrally mediated sympathetic control of circulation may be lacking in the lower part of the body as a result of the disrupted spinal cord. Theoretically, this would lead to vasodilatation of peripheral vessels and, therefore, to a decrease in vascular resistance below the lesion. However, the part of the body below the spinal lesion is paralyzed and extremely inactive, which may affect the vascular properties in this part of the body as well [7]. There are several reasons to hypothesize that vascular resistance in the legs of long- term spinal-cord-injured individuals will be increased: (1) animal research has shown that endothelial function changes after long-term sympathectomy with a predominance of endothelin- 1 release and a decrease in nitric oxide release [8]. (2) As a result of deconditioning of the leg muscles, oxygen demand will be low, and oxygen delivery will be geared accordingly, which would lead to vascular atrophy, most likely via flow-dependent mechanisms [9]. (3) In long-term injury patients, the clinically obvious cold and blue-colored legs and the reported poor wound healing suggest reduced leg blood flow and possibly increased vascular resistance [10]. The loss of autonomic control and the lack of movement induce dramatic adaptations in the central and peripheral circulatory system in the spinal-cord injury population [11]. Important structural and functional remodeling in the peripheral vascular system of paralyzed limbs has been reported, including a reduced diameter of the arteries and arterial stiffness. The authors agreed to this hypothesis of increase in the peripheral resistance. Blood stasis leads to thrombosis susceptibility, delayed wound healing, and increased risk of pressure sore formation, and brings about lymphedema $[12,13]$. This paraplegic state could imply a preconditioned and susceptible state for Raynaud's phenomenon, able to be provoked by $\beta$-blocker medication. To date, there has been no experimental study on the prevalence of Raynaud's phenomenon or cold sensation in the feet of long-term paraplegic patients who have taken diuretics medication, though some reports show that hydrochlorothiazide could aggravate Raynaud's phenomenon [2, 14].

When the symptom of reduced toe blood flow is acute or diagnosed early, the cessation of thiazide medication can be enough to stop progression of skin necrosis. Otherwise, the use of calcium channel blockers can be helpful to increase the circulation of peripheral vessels, even if there is arterial stiffness in the lesion (Fig. 4). Calcium

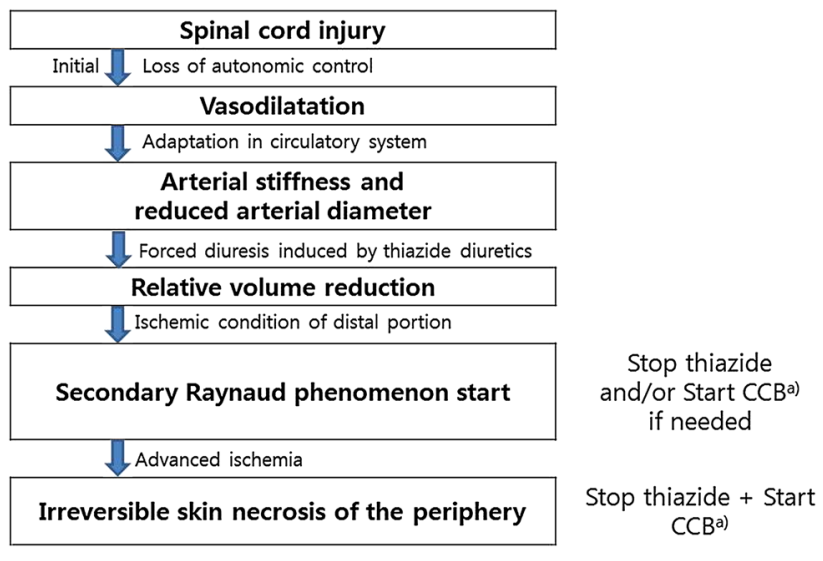

Fig. 4 Schematic flow of the relationship between thiazide diuretics and secondary Raynaud's phenomenon in paraplegic patients with hypertension. $C C B$ calcium channel blocker 
antagonists have become very popular and their use has been extended to additional conditions such as angina pectoris, hypertrophic cardiomyopathy, Raynaud's phenomenon, and cerebral vasospasm [15]. This calcium channel blocker could then release vasoconstriction.

\section{Conclusion}

If a hypertensive patient with paraplegia complains of unstable aggravating wounds on toes, secondary Raynaud's phenomenon should be considered, especially if the patient is receiving diuretics.

Funding This work was supported by the sabbatical research grant of the corresponding author from Daegu Catholic University in 2016-2017.

\section{Compliance with Ethical Standards}

Conflict of interest The authors, Yong Jig Lee and Kisoo Park have no competing interests to declare.

Informed consent Written informed consent was obtained from the two patients involved, for publication of the details included in this article and for inclusion of the accompanying images. Copies of the written consent statements may be requested for review from the corresponding author.

Open Access This article is distributed under the terms of the Creative Commons Attribution-NonCommercial 4.0 International License (http://creativecommons.org/licenses/by-nc/4.0/), which permits any noncommercial use, distribution, and reproduction in any medium, provided you give appropriate credit to the original author(s) and the source, provide a link to the Creative Commons license, and indicate if changes were made.

\section{References}

1. Joel AB, Winston S. Raynaud's phenomenon: pathogenesis and management. J Am Acad Dermatol. 2008;59:633-53.

2. eHealthMe. Review: could Hydrochlorothiazide cause Raynaud's phenomenon aggravated? [Internet]. eHealthMe.com. http:// www.ehealthme.com/ds/hydrochlorothiazide/raynaud-sphenomenon. Cited 4 July 2014.

3. Mammen EF. Pathogenesis of venous thrombosis. Chest. 1992;102:640S-4S.

4. Nigrovic PA, Fuhlbrigge RC, Sundel RP. Raynaud's phenomenon in children: a retrospective review of 123 patients. Pediatrics. 2003;111:715-21.

5. Lim MJ, Kwon SR, Jung KH, et al. Digital thermography of the fingers and toes in Raynaud's phenomenon. J Korean Med Sci. 2014;29:502-6.

6. Frank LU. Raynaud's phenomenon. Hosp Phys. 2001;37:27-30.

7. Hopman MT, Groothuis JT, Flendrie M, et al. Increased vascular resistance in paralyzed legs after spinal cord injury is reversible by training. J Appl Physiol. 2002;93:1966-72.

8. Aliev G, Ralevic V, Burnstock G. Depression of endothelial nitric oxide synthase but increased expression of endothelin-1 immunoreactivity in rat thoracic aortic endothelium associated with long-term, but not short-term, sympathectomy. Circ Res. 1996;79:317-23.

9. Hopman MT, van Asten WN, Oeseburg B. Changes in blood flow in the common femoral artery related to inactivity and muscle atrophy in individuals with long-standing paraplegia. Adv Exp Med Biol. 1996;388:379-83.

10. Basson MD, Burney RE. Defective wound healing in patients with paraplegia and quadriplegia. Surg Gynecol Obstet. 1982;155:9-12.

11. Jacobs PL, Nash MS. Exercise recommendations for individuals with spinal cord injury. Sports Med. 2004;34:727-51.

12. Wigley FM. Clinical practice: Raynaud's phenomenon. N Engl J Med. 2002;347:1001-8.

13. Walden R, Bass A, Ohry A, et al. Pulse volume recording disturbances in paraplegic patients. Paraplegia. 1991;29:457-62.

14. Coffman JD. Raynaud's phenomenon. Oxford: Oxford University Press; 1989. p. 72.

15. Grossman E, Messerli FH. Calcium antagonists. Prog Cardiovasc Dis. 2004;47:34-57. 